\title{
Spatial Peak Shift and Generalization in Pigeons
}

\author{
Ken Cheng \\ Macquarie University \\ Marcia L. Spetch and Michael Johnston \\ University of Alberta
}

\begin{abstract}
How pigeons generalize across spatial locations was examined in the 4 experiments reported in this article. During training, a square was presented at a fixed height at 1 of 2 horizontal locations on a monitor screen. One location $(S+)$ signaled reward, whereas the other one $(S-)$ signaled no reward. The birds were then tested occasionally with a range of locations. After training with $S+$ only, the generalization gradient peaked at $S+$ and was approximately Gaussian in shape. After training with equal numbers of $S+$ and $S-$ trials, response rates were higher on the $S+$ side of the distribution. This asymmetry diminished over testing. When the $S+$ and $S-$ were close together, the peak of responding was shifted on initial generalization tests. Generalization gradients along the orthogonal vertical dimension were approximately exponential in shape. This is the first demonstration of generalization and peak shift in the spatial domain.
\end{abstract}

An important task for the experimental analysis of behavior has been to understand how stimuli present when behavior is reinforced to gain control over the behavior. Stimulus generalization-responding in a similar fashion despite changes in properties of a stimulus-and discrimination-responding differently when a stimulus property is changed-are opposite ends of a continuum of stimulus control. Stimulus control typically is indexed by decremental stimulus generalization gradients, in which increasing changes in a stimulus value lead to increasing changes in responding.

Guttman and Kalish (1956) conducted a now-classic study of stimulus generalization in pigeons. They reinforced pigeons on an intermittent schedule for pecking a key in the presence of a monochromatic light source. Different groups of pigeons were trained with different spectral stimuli. After a steady rate of responding emerged, the pigeons were tested in extinction with a range of spectral wavelengths on either side of, and including, the training value. Plots of responses as a function of spectral wavelength showed orderly generalization gradients, with the highest rate of responding at the

Ken Cheng, School of Behavioural Sciences, Macquarie University, Sydney, Australia; Marcia L. Spetch and Michael Johnston, Department of Psychology, University of Alberta, Edmonton, Alberta, Canada.

This research was supported by grants from Macquarie University and the Australian Research Council and a Natural Sciences and Engineering Council of Canada research grant. Some of these data were collected as part of the thesis requirement for an honors bachelor of science degree in psychology by Michael Johnston.

We thank D. Kelly and S. Reid for assistance in conducting the research.

Correspondence concerning this article should be addressed either to Ken Cheng, School of Behavioural Sciences, Macquarie University, Sydney NSW 2109 Australia, or to Marcia L. Spetch, Department of Psychology, University of Alberta, Edmonton, Alberta, Canada T6G 2E9. Electronic mail may be sent via Internet either to kcheng@bnnyip.bhs.mq.edu.au or to mspetch@psych. ualberta.ca. training value and increasingly lower rates of responding at increasingly different test values.

Since then, a great deal of research has been directed at investigating factors that influence the steepness and form of stimulus generalization gradients. One general finding has been that generalization gradients become steeper if discrimination training includes periods of reinforced responding in the presence of the training stimulus (referred to as the positive stimulus, or $S+$ ) randomly alternated with periods during which responding is not reinforced in the presence of a different stimulus (referred to as the negative stimulus, or $S-$ ). Discrimination training can be either intradimensional, in which the $\mathbf{S}+$ and $\mathbf{S}$ - differ only along the dimension on which generalization tests will be conducted, or interdimensional, in which the $\mathbf{S}+$ and $\mathbf{S}-$ differ in many stimulus dimensions. For example, Jenkins and Harrison (1960) conducted tonal frequency generalization tests in pigeons following (a) nondifferential training in the presence of a $1000-\mathrm{Hz}$ tone; (b) interdimensional training, in which the $\mathrm{S}+$ was a $1000-\mathrm{Hz}$ tone and the $\mathrm{S}-$ was silence; or (c) intradimensional training, in which the $\mathrm{S}+$ was a $1000-\mathrm{Hz}$ tone and the $\mathrm{S}-$ was a $950-\mathrm{Hz}$ tone. The generalization gradient was steepest for birds given intradimensional training and flattest for birds given nondifferential training.

Intradimensional discrimination training not only sharpens the generalization function but also can shift the location of peak responding toward a value away from the $S-$. In the classic demonstration of this peak-shift effect, Hanson (1959) gave four groups of pigeons intradimensional discrimination training on the wavelength dimension. The $S+$ for all groups was $550 \mathrm{~nm}$, and the $S-$ was $555,560,570$, or 590 $\mathrm{nm}$. A control group received nondifferential training with the $\mathbf{S}+$ only. After training, all groups were given generalization tests with stimuli ranging from 480 to $620 \mathrm{~nm}$. Only the control group showed a peak of responding at the $S+$. The other groups responded more to wavelength values below the $S+$, with the size of the peak shift varying inversely with the magnitude of the difference between the $\mathrm{S}+$ and $\mathrm{S}-$ values. 
The peak-shift effect has since been demonstrated many times, and most subsequent research has supported Hanson's (1959) finding that the magnitude of the peak-shift effect increases as the spacing between the $\mathrm{S}+$ and $\mathrm{S}-$ decreases. Moreover, the generality of the peak-shift effect has been extended to a variety of stimulus dimensions, including visual intensity (Ernst, Engberg, \& Thomas, 1971), auditory intensity (Thomas \& Setzer, 1972), line tilt (Bloomfield, 1967), floor tilt (Riccio, Urda, \& Thomas, 1966), object size (Dougherty \& Lewis, 1991), and even relative numerosity (Honig \& Stewart, 1993). The effect has also been extended to various species including goldfish (Ames \& Yarczower, 1965), rats and guinea pigs (Thomas \& Setzer, 1972), chickens (Rudolph \& Honig, 1972), horses (Dougherty \& Lewis, 1991), and humans (Doll \& Thomas, 1967). Thus, the peak-shift effect appears to be a reliable and general result of intradimensional discrimination training, although individual differences do exist, and some individuals fail to show the effect (see Honig \& Urcuioli, 1981; Rilling, 1977; Purtle, 1973, for reviews). Even in cases in which a shift in modal responding does not occur, however, intradimensional discrimination training typically results in asymmetrical generalization gradients in which animals respond more to values on the $S+$ side of the distribution than to values on the S- side, an effect that is referred to as an area shift (Rilling, 1977).

The peak-shift effect has been the source of considerable theoretical interest. The predominant theoretical framework in which the effect has been discussed is Spence's (1937) theory of discrimination learning, in which an excitatory gradient around the $S+$ and an inhibitory gradient around the $S-$ are assumed to form during discrimination training. The interaction of these two gradients, specifically their algebraic summation, is thought to displace the peak of the response distribution away from the $\mathbf{S}+$ in the direction opposite the S-. Spence's theory is an example of an "absolute" approach to discrimination learning because it assumes that organisms learn response tendencies to specific stimulus values. An alternative approach has been to assume that organisms learn about relationships between the stimulus values. Recently, Thomas and colleagues (e.g., Thomas, 1974; Thomas, Mood, Morrison, \& Wiertelak, 1991) proposed a relational interpretation of the peak-shift effect, on the basis of principles derived from Helson's (1964) adaptation-level theory. They suggested that the average value of stimuli presented during intradimensional discrimination training forms an adaptation level, or frame of reference, and that organisms learn to respond to a value that is $X$ units above or below this adaptation level. During generalization testing without feedback, the adaptation level becomes the average of the range of values used in testing. If this range differs from that used in training, then the code for $S+$ corresponds to a different stimulus value, in absolute terms, from the $S+$ value during training. The rule of responding $X$ units away from the adaptation level will lead to a peak-shift effect. There is now considerable evidence, primarily from studies conducted on humans, that supports the adaptationlevel interpretation of the peak-shift effect. For example, shifts in the peak toward the $S-$ have been found when the test values presented are centered at a value that is closer to the S - than to the S+ (Thomas et al., 1991). The peak of responding should depend crucially on the range of stimulus values used on tests, and Thomas et al. found those predicted range effects. On the other hand, adaptation-level effects are less forthcoming in research with nonhuman animals, perhaps because the extensive discrimination training typically provided creates a more stable adaptation level that is less likely or slower to change when new values are introduced during testing (Thomas et al., 1991). The robust peak shift that often occurs under the experimental parameters that seem resistant to adaptation-level effects makes it unlikely that adaptation level underlies the peak-shift effect typically seen in nonhuman animals. Nevertheless, the potential for range effects should be considered in all studies of peak shift.

The present research was designed to extend the study of stimulus generalization and the peak-shift effect to a new stimulus dimension; namely, spatial location. Spatial location is a functionally very important dimension in the lives of most animals. An ability to encode and remember the location of important stimuli, such as sources of food or homes, is often critical to survival. Not surprisingly, a great deal of research has been devoted to understanding the mechanisms used by animals to remember and locate important places (e.g., see Cheng \& Spetch, in press; Gallistel, 1990, for reviews). Although some aspects of spatial learning suggest specialized processes (e.g., Cheng, 1986), general principles in the processing of spatial information have also been revealed (e.g., Cheng, 1992), and spatial learning seems to follow some of the same general principles of learning derived from research within traditional classical and operant conditioning procedures (see Cheng \& Spetch, in press; Spetch, 1995). As yet, however, generalization along the spatial dimension has not systematically been explored. It is of interest to determine whether animals' responses to variations in the spatial location of a stimulus reveal generalization functions and peak-shift effects that are typical of their responses to variations in properties of the stimulus itself.

The first three experiments presented here were designed to explore generalization and the peak-shift effect in the spatial dimension. In these experiments, pigeons were reinforced on a fixed-interval (FI) schedule for pecking at a computer screen following presentation of a small yellow square in a fixed screen location ( + ). In Experiment 1, all training trials consisted of $S+$ presentations. In Experiments 2 and 3 , trials with $S+$ were mixed randomly with trials in which the same stimulus was presented at a fixed but horizontally different location, and responding was not reinforced (S-). In each case, training was followed by generalization tests in which occasional unreinforced probe trials presented the square at 1 of 11 horizontal locations, including and extending beyond the training locations. Experiments 2 and 3 differed only in the size and spacing of the training and test stimuli.

In all three experiments, we tested the same birds in different phases, with different ranges of test stimuli. In one series, we used all locations on the horizontal dimensions. In 
two other series, we used partial ranges on the $S+$ and $S-$ sides, respectively. If range effects are found, the tests with partial ranges should shift the peak of responding, toward the side on which the test stimuli are found. We did not expect such range effects. The reason is that testing was done with occasional unrewarded trials mixed with continuing training trials. The training trials should serve to anchor the adaptation level, a factor we wanted to control for in testing. Lack of range effects here thus should not be taken as evidence against Thomas's (1974) adaptation-level hypothesis.

Experiment 4 provided a preliminary exploration of the scaling of space in generalization. On the basis of data from multidimensional scaling of errors in a spatial memory task, Wilkie (1989) concluded that pigeons possessed a linear scale of space with a Euclidean metric. If we therefore assume a linear scale, we can find out what form the generalization function is in psychological space. For this purpose, we used the generalization data already collected in Experiment 1, and we conducted generalization tests in the vertical spatial dimension with the birds that had participated in Experiments 2 and 3.

\section{Experiment 1}

\section{Method}

\section{Animals}

Three adult silver king pigeons (Columba livia) with varied experimental histories, but no previous experience in tasks in which the touch screen was used, were used in this experiment. The birds were maintained at approximately $85 \%$ of their free-feeding weights by mixed grain obtained during experimental sessions and supplemental feedings of pigeon chow (Kaytee Pigeon Pellets, Katee Products, Chilton, WI). They were housed in large individual cages under a 12-hr light-dark cycle (lights on at 6:00 a.m.). Water and grit were freely available in the home cages.

\section{Apparatus}

The experimental chamber was a custom built enclosure, $44 \times$ $32 \times 74 \mathrm{~cm}$ (inside dimensions). A $28-\times 20-\mathrm{cm}$ monitor opening in the center of the back wall, $10 \mathrm{~cm}$ above the floor, provided access to a color monitor (Zenith 1492, St. Joseph, MI) equipped with an infrared touch frame (Carroll Touch, 1492 Smart Frame, Round Rock, TX). A thin sheet of Plexiglas covered the monitor screen, and spacers were used to recess the touch frame by approximately $3 \mathrm{~cm}$ from the monitor opening and to separate the frame from the monitor by approximately $1.5 \mathrm{~cm}$. Two Gerbrands (Arlington, MA) feeders filled with mixed grain were located on the back wall: one $8 \mathrm{~cm}$ to the left and one $8 \mathrm{~cm}$ to the right of the monitor opening. Lamps in each feeder illuminated feeder presentations, and photocells measured head entries into the hopper. All food deliveries consisted of 2-s access to one of the two feeders, selected randomly on each trial so as to minimize bias toward a particular side of the screen. Microcomputers in an adjacent room controlled experimental contingencies and recorded peck coordinates. The touch frame was programmed to detect individual pecks (i.e., detection of a beam break, then a return to unbroken beams before another peck would be recorded).

\section{Search Space and Stimuli}

The search space was the illuminated surface of the color monitor, approximately $25 \times 19 \mathrm{~cm}$. The discriminative stimulus was a yellow patch, approximately $2-\mathrm{cm}$ square, displayed on a dark gray background. Placement of the stimulus was determined by pixel location, with the entire screen comprising a $640-\times 480-$ pixel area (approximately 25 pixels per $\mathrm{cm}$ ). During training, the $S+$ was centered 270 pixels from the left edge of the screen (i.e., 50 pixels left of center) for 1 bird and 370 pixels from the left edge of the screen (i.e., 50 pixels right of center) for the other birds. In either case, the $S+$ was centered 200 pixels from the top of the screen. Figure 1 shows a schematic of the search space, the locations used for the training stimulus, and the locations used during subsequent generalization testing. The warning stimulus consisted of a uniform illumination of the entire screen with white. The screen was dark during the intertrial interval (ITI).

\section{Procedure}

Each bird initially received one or more preliminary training sessions with an autoshaping procedure to establish pecking. During autoshaping trials, the $S+$ was presented for $8 \mathrm{~s}$ or until the pigeon pecked anywhere on the screen. The trial ended with food whether or not the bird pecked and was followed by a 40-s ITI. This preliminary training continued until the bird pecked on at least $80 \%$ of the trials in a session.

Training. Each bird next received 25 training sessions, each consisting of 120 trials separated by $2-s$ ITIs. On each trial, the warning stimulus was presented until the bird made a single peck anywhere on the screen, and then the $S+$ was presented. Presentation of the S+ initiated a 5-s FI schedule in which the first peck made anywhere on the screen after $5 \mathrm{~s}$ terminated the display and produced food.

Each bird then received two test phases, separated by a minimum of two sessions of baseline training.

Generalization Test 1: Full distribution. This test consisted of eight sessions during which 33 test trials were interspersed randomly among $84 \mathrm{~S}+$ baseline trials. On test trials, the horizontal location of the discriminative stimulus was varied. The test locations were centered at $70,120,170,220,270,320,370,420$,

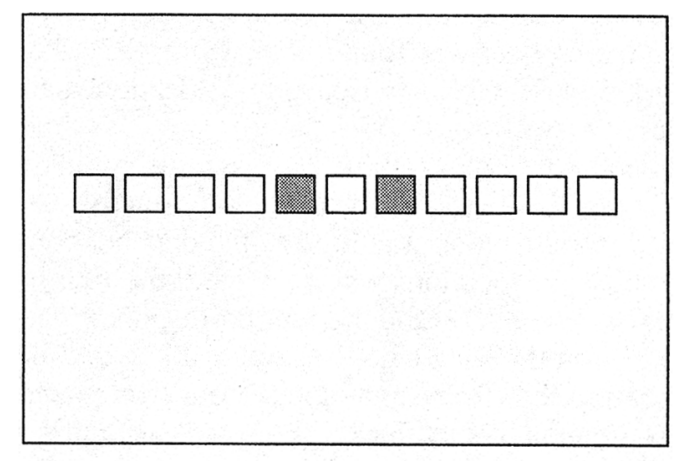

Training Stimulus $\square$ Test Stimulus

Figure 1. Schematic diagram showing the locations of the training stimuli and test stimuli on the computer screen in Experiments 1 and 2. In Experiment 1, each bird was trained with only one of the two training stimuli. In Experiment 2, one of the training stimuli served as the $S+$, and one served as the $S-$. 
470,520 , and 570 pixels from the left edge of the screen. Each test location was presented three times in each session. Test stimulus presentations initiated a 5-s fixed time (FT) that ended without food. Baseline trials were the same as during training. Two sessions of baseline training were interspersed between the fourth and fifth test sessions.

Generalization Tests 2 and 3: Partial distributions. During these two series, generalization tests included partial rather than full distributions. Eight sessions of testing were conducted for the left side of the distribution, and eight sessions were conducted for the right side of the distribution. In each session, test stimuli were presented at 7 of the 11 locations used in the first test phase. During Series 2, the 7 locations included the four places on the short side of the $\mathrm{S}+$ and extended to two places on the long side (i.e., the 7 right-most locations for 2 birds and the 7 left-most locations for 1 bird). During Series 3, the 7 locations consisted of the $S+$ and the 6 locations on the long side of the S+ (i.e., the right side of the distribution for 1 bird and the left side for 2 birds). In each session, 21 test trials (3 of each location) were interspersed among 84 baseline $S+$ trials.

In this and subsequent experiments, the alpha level used for statistical significance was .05 .

\section{Results}

Rates of responding as a function of location are presented in Figure 2. Locations are coded with respect to the short and long sides of $\mathbf{S}+$, which amounts to reflecting the data for some birds around the middle to make $S+$ for every bird coincide. Two types of statistical tests were conducted by using within-subject analysis of variance (ANOVA). In one test, the eight sessions of tests in each series were divided into four blocks to make a block factor. Location was the other factor, with all locations included. Absolute rate of responding was the dependent measure. The ANOVA for Series 1 (full distribution of locations) revealed a significant block effect, $F(3,9)=6.20$, a location effect, $F(10,30)=15.63$, and a Block $\times$ Location interaction, $F(30,90)=1.93$. Inspection of the data showed that the interaction and block effects were attributable mainly to the first block, which showed a shallower gradient than the others. When analyzing data from Blocks $2-4$ only, both effects disappeared. For Series 2 (short side of $S+$ ), only a location main effect was found, $F(6,18)=9.39$. For Series 3 (long side of $\mathrm{S}+$ ), likewise, only a location main effect was found, $F(6,18)=19.57$.

A second ANOVA examined the symmetry of results in Series 1 . For this test, rate of responding relative to $S+$ for each bird in each block was used as the dependent measure. Only a subset of locations was examined: the four locations on the short side of $\mathrm{S}+$ and the four corresponding locations next to $\mathbf{S}+$ on the long side. $\mathbf{S}+$ was excluded. Side (two), distance from $\mathrm{S}+$ (four), and blocks (four) were factors. The ANOVA found a block effect, $F(3,9)=7.91$, and a distance effect, $F(3,9)=43.89$. The lack of a side effect and a Side $X$ Distance interaction indicates that the generalization gradient was approximately symmetric about $\mathrm{S}+$.

\section{Discussion}

The results of this experiment show a generalization gradient along a spatial dimension much like what is

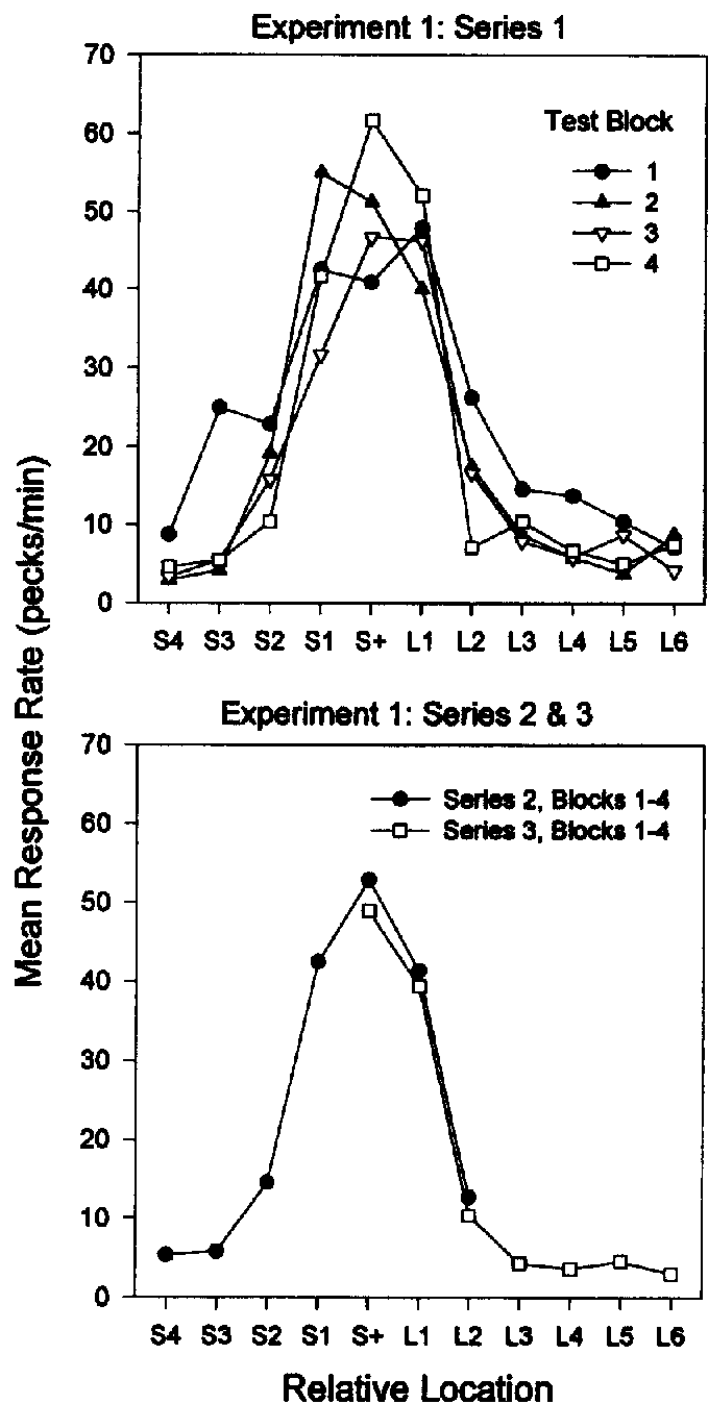

Figure 2. Generalization gradients from Experiment 1. Top panel: Series 1, full distribution of locations. Bottom panel: Series 2 and 3, partial distribution of locations. S stands for short side of $\mathrm{S}+, \mathrm{L}$ stands for long side of $\mathrm{S}+$.

typically found for generalization along other dimensions. Responding peaked at the $S+$ value and decreased symmetrically with distance from $\mathrm{S}+$. Tests with partial distributions of locations still found a peak at $S+$, giving no evidence for range effects.

\section{Experiment 2}

This experiment was designed to investigate the effect of discrimination training on stimulus control by location. This experiment was similar to Experiment 1 except that training involved a discrimination procedure between a positive location $(\mathrm{S}+)$ and a negative location $(\mathrm{S}-$ ). The same generalization tests conducted in Experiment 1 were presented following training. 


\section{Method}

\section{Animals}

This experiment was conducted with four adult silver king pigeons that had extensive experimental histories, including tasks conducted on the touch screen. None of the birds had prior experience with spatial successive discriminations. The birds were housed and maintained as in Experiment 1.

\section{Apparatus, Search Space, and Stimuli}

These were identical to those described in Experiment 1.

\section{Procedure}

The locations of the positive and negative discriminative stimuli ( $S+$ and $S^{-}$) were counterbalanced across birds. For 2 birds, the $\mathbf{S}+$ was 270 pixels from the left edge, and the $S-$ was 370 pixels from the left edge, whereas the opposite arrangement was used for the remaining 2 birds.

Preliminary training was identical to that described in Experiment 1. Training procedures were similar to those described in Experiment 1 except for the inclusion of $S-$ trials. In each training session, $\mathbf{S}+$ and $\mathbf{S}-$ trials were scheduled to occur equally often in a randomly determined order. $S+$ trials were the same as in Experiment 1. On $S-$ trials, presentation of the yellow square at the S- location initiated a 5-s FT period followed by termination of the display, and no food was delivered.

Discrimination ratios (DRs) were calculated for each session on the basis of pecks made during the first $5 \mathrm{~s}$ of $\mathrm{S}+$ and $\mathrm{S}-$ presentations. The total number of pecks made during $S+$ presentations was divided by the sum of the total number of pecks made during $S+$ presentations and the total number of pecks made during $S-$ presentations. A DR of 1 represents perfect discrimination (responding only on $\mathrm{S}+$ trials), and a DR of .5 represents no discrimination (responding equally on $S+$ and $S-$ trials).

Each bird remained in training for a minimum of 28 sessions and until its average discrimination ratio over the preceding 4 sessions was above .75 . Each bird then received three series of generalization tests, which were identical to those described in Experiment 1 except that baseline trials consisted of a mixture of $\mathrm{S}+$ and $\mathrm{S}-$ trials.

\section{Results}

Three birds met the accuracy criterion within the minimum 28 sessions, and the 4th bird met the criterion on Session 32. Rates of responding as a function of location during generalization testing are presented in Figure 3. For 2 birds, their data have been reflected about the middle to make the $\mathrm{S}+$ locations for all birds coincide. With the full range of test locations in Series 1, no peak shift was found, but a noticeable area shift was found. This was short lived and had largely subsided by Block 3 of testing. In Series 2 (locations on S+ side), some area shift was found in the first block. This effect also appeared to subside quickly.

The ANOVA on absolute rates in Series 1 revealed a block effect, $F(3,9)=4.26$, a location effect, $F(10,30)=21.55$, and a Block $\times$ Location interaction, $F(30,90)=2.45$. By Block 3, results appear stable. Indeed, a test on Blocks 3 and 4 only revealed no interaction. In Series 2 , a location effect

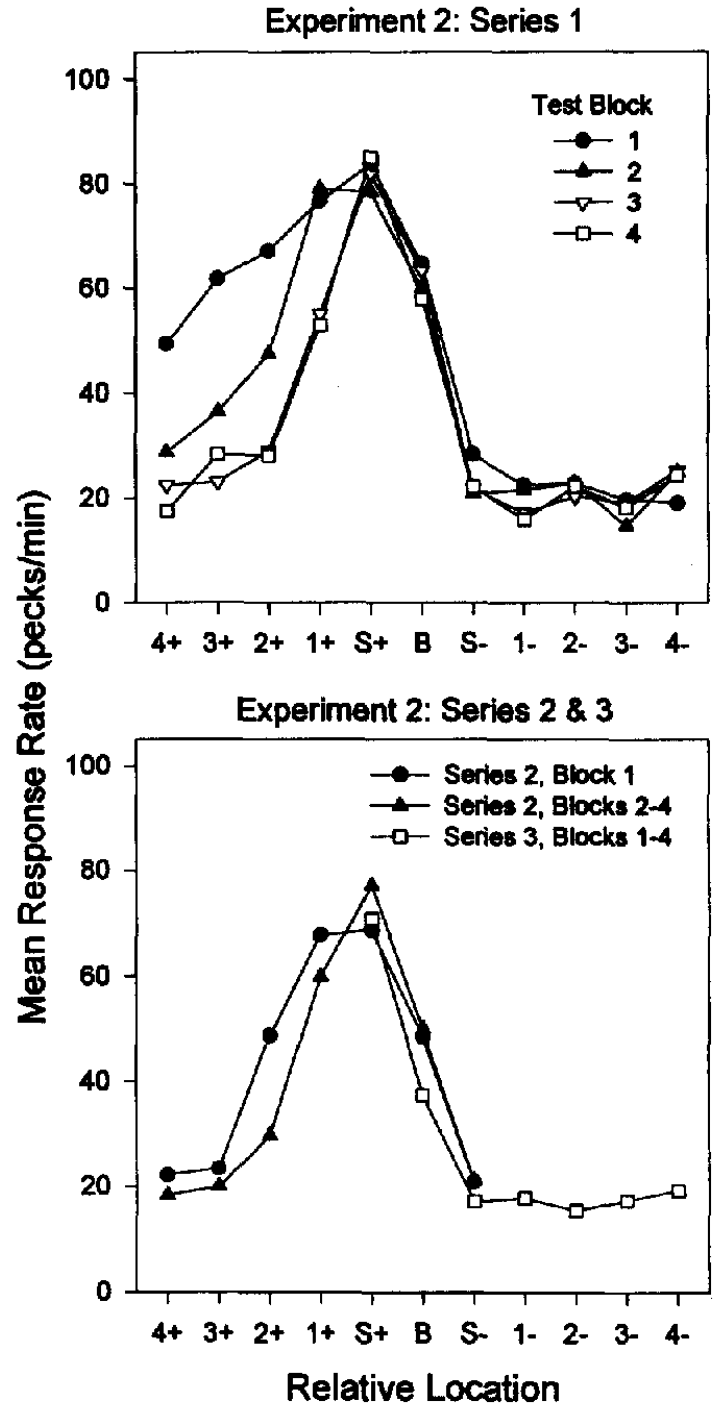

Figure 3. Response rates as a function of location in Experiment 2. Top panel: Series 1, full distribution of locations. Bottom panel: Series 2 and 3, partial distribution of locations. Labels $1+$ to $4+$ refer to values at increasing distances on the $S+$ side, and $1-$ to 4are values at increasing distances on the $S-$ side. $B$ indicates location between the $S+$ and $S-$.

was found, $F(6,18)=6.19$, as was a Block $\times$ Location interaction, $F(18,54)=1.90$. The interaction was attributable mainly to the first block, as it disappeared if only the data from Blocks $2-4$ were examined. In Series 3, only a location main effect was found, $F(6,18)=6.11$.

The symmetry test on Series 1 (analogous to the test for Experiment 1) revealed a main effect of block, $F(3,9)=$ 4.08, a main effect of distance from $\mathrm{S}+, F(3,9)=89.67$, a Block $\times$ Side interaction, $F(3,9)=4.67$, and, most crucially, a Side $\times$ Distance From $S+$ interaction, $F(3,9)=$ 5.83. This last effect showed asymmetry about $S+$ in response rates, confirming the area shift effect. The Block $X$ Side interaction confirmed impressions that the area shift subsided with testing. Indeed, a symmetry test on the data 
from Blocks 3 and 4 revealed only an effect of distance from $\mathrm{S}+, F(3,9)=35.43$, thus indicating a symmetric gradient.

\section{Discussion}

The peak-shift manipulation, training with $S-$ at 100 pixel distance (approximately $4 \mathrm{~cm}$ ) from $S+$, produced not a peak shift but an area shift. The gradient became asymmetric, falling less steeply on the $\mathrm{S}+$ side. The effect was short lived, as the gradient became symmetrical by the third block of testing. Tests with different ranges of stimulus values again all resulted in peaks of responding at $S+$, giving no evidence of range effects.

We think that the drop in responding over tests on the $\mathrm{S}+$ side stems from our procedure of occasional testing mixed with continued training at $\mathrm{S}+$ and $\mathrm{S}-$ locations. This taught the birds that while $S+$ was often rewarded, other locations on the $\mathrm{S}+$ side were never rewarded. In short, the birds were learning to discriminate locations on the $S+$ side because of the contingencies of reward during testing. In the General Discussion, we present a mechanistic model to account for this learning.

\section{Experiment 3}

In studies of peak shift with other stimulus dimensions, spacing of the $\mathbf{S}+$ and $\mathbf{S}-$ is an important factor. Specifically, a peak shift is more likely to occur if the $S+$ and $S-$ are close together. For this experiment, we therefore decreased the distance between locations.

\section{Method}

\section{Animals}

Four adult silver king pigeons served in this experiment. They were housed and maintained as described in Experiment 1. All pigeons had extensive experimental histories, including experience in touch-screen search tasks, but none had previously served in a spatial successive discrimination task.

\section{Apparatus}

The experimental chamber was a modified BRS/LVE (Laurel, MD) operant chamber with a $28-\times 20$-cm monitor opening in the back wall, $9 \mathrm{~cm}$ above the floor. The chamber contained two BRS/LVE grain hoppers, one on each of the side walls, $8 \mathrm{~cm}$ from the back wall. The monitor, touch frame, and other aspects of the apparatus were the same as those described in Experiment 1.

\section{Search Space and Stimuli}

The search space and all aspects of the stimulus display were identical to those described in Experiment 1, with two exceptions: (a) the discriminative stimuli were smaller (approximately $1.2-\mathrm{cm}$ square), and (b) placement of the discriminative stimuli differed. During training, the centers of the discriminative stimuli were located 240 pixels from the top of the screen. One discriminative stimulus was centered at 290 pixels, and the other was centered at 350 pixels from the left edge of the screen (i.e., each 30 pixels from the center of the screen).

\section{Procedure}

For 2 birds, the $S+$ was 290 pixels and the $S-$ was 350 pixels from the left edge of the screen, whereas the opposite arrangement was used for the other 2 birds. The training procedures and the criteria for completion of training were the same as in Experiment 2, except that the accuracy criterion was lowered to $70 \%$ for 1 bird that failed to meet the $\mathbf{7 5 \%}$ criterion within $\mathbf{4 5}$ sessions. After the training, each bird received three generalization test series that were identical to those described in Experiment 1 except for the spacing of the test stimuli. In this experiment, the test stimuli were at $170,200,230,260,290,320,350,380,410,440$, or 470 pixels from the left edge of the screen (all 240 pixels from the top of the screen).

\section{Results}

Two birds met the accuracy criterion within the minimum 28 sessions, 1 bird met it on Session 35, and the 4th bird met the reduced criterion on Session 49. Rates of responding as a function of location are shown in Figure 4, again with the data for 2 birds reflected about the middle to make $S+$ for all birds coincide. A noticeable peak-shift effect was found in this experiment. The effect appears to subside with testing and, by Series 2, had become an area shift rather than a peak shift.

The ANOVA on absolute rates in Series 1 found a block effect, $F(3,9)=12.02$, a location effect, $F(10,30)=17.05$, but no interaction. For Series 2 (S+ side), a location effect, $F(6,18)=5.43$, and a Block $\times$ Location interaction, $F(18$, $54)=2.05$, were found. This interaction supports the impression that the peak-shift effect subsided with testing. In Series 3 (S- side), only a location main effect was found, $F(6,18)=9.30$.

The symmetry test on Series 1 found many effects: a side effect, $F(1,3)=72.69$, indicating asymmetry, a distance from $\mathrm{S}+$ effect, $F(3,9)=39.61$, and a Side $\times$ Distance From $S+$ interaction, $F(3,9)=21.06$, also indicating asymmetry. It also found a Block $\times$ Distance interaction, $F(9,27)=2.70$, and a three-way interaction, $F(9,27)=$ 2.51. These interactions indicate that the nature of the asymmetry was changing over test blocks, confirming the impressions that the peak shift was subsiding with testing.

\section{Discussion}

In this experiment with smaller distance between $S+$ and $\mathrm{S}-$, the peak-shift effect was stronger than in Experiment 2. An actual peak shift was found early in testing. This subsided to an area shift effect by the end of testing in Series 2. In conjunction with the results of Experiment 2, these results show that peak shift is stronger when $S-$ is closer to $\mathrm{S}+$, a pattern found with other stimulus dimensions. In both experiments, however, the peak- or area shift effect subsided with testing.

Once again in this experiment, no range effects were found. If anything, the subsiding peak-shift effect in Series 2 put the peak closer to $S+$, which is in the wrong direction predicted by range effects. It appears that our manipulations have successfully controlled for range effects. 


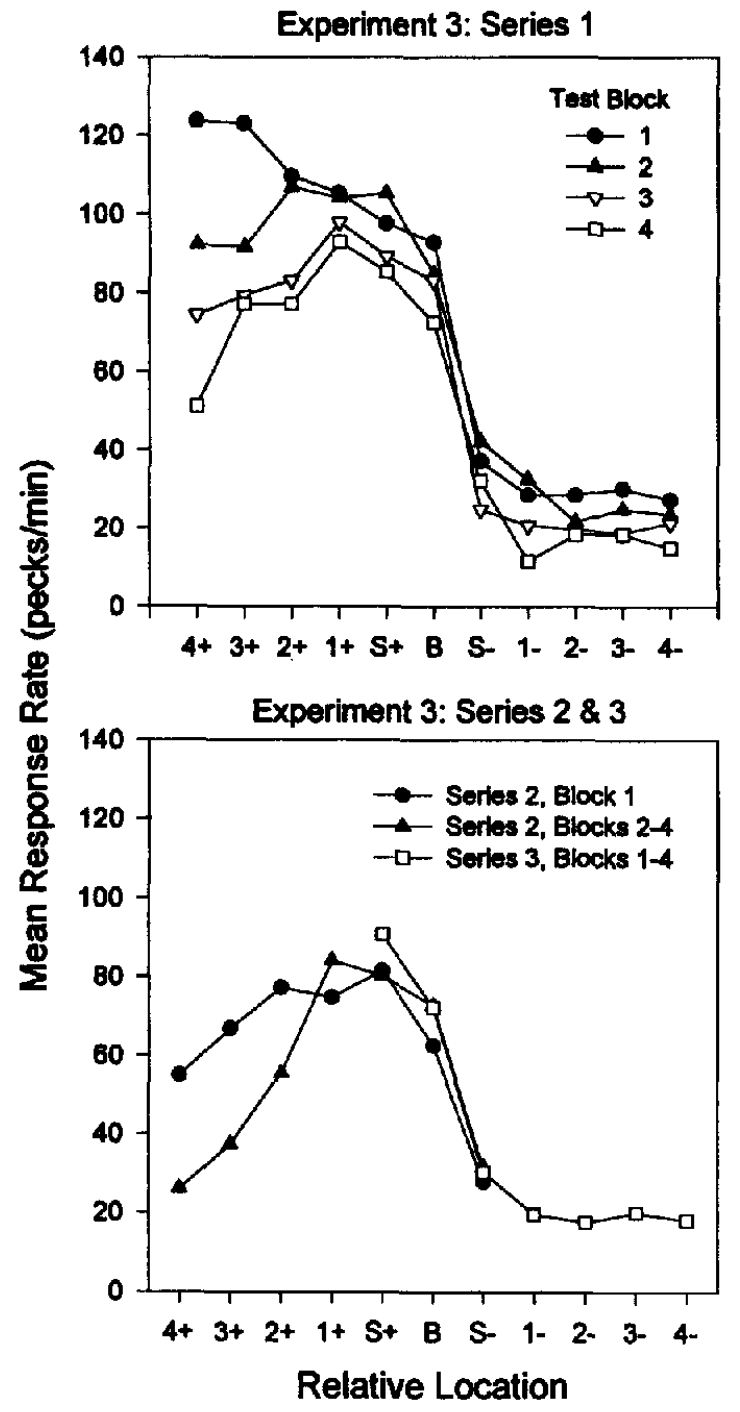

Figure 4. Response rates as a function of location in Experiment 3. Top panel: Series 1, full distribution of locations. Bottom panel: Series 2 and 3, partial distribution of locations. Labels $1+$ to $4+$ refer to values at increasing distances on the $S+$ side, and $1-$ to 4are values at increasing distances on the $\mathrm{S}-$ side. $\mathrm{B}$ indicates location between the $S+$ and $S-$.

\section{Experiment 4}

This experiment is a continuation of the other three, with the purpose of examining the scaling of space in generalization. The horizontal generalization data in Experiment 1 were used, but the data from Experiments 2 and 3 were problematic for the present purpose because one of the locations was presented many times as $\mathbf{S}$ - in the peak-shift paradigm. We therefore obtained further data on generalization by conducting tests along the vertical dimension with the birds from Experiments 2 and 3. With these generalization data, we could examine the form of the generalization function.

On the basis of data from multidimensional scaling of errors in a spatial memory task, Wilkie (1989) concluded that pigeons possessed a linear and Euclidean psychological scale of space. What is meant by linear is that one linear unit distance in physical space corresponds to one unit distance in psychological space. Other possibilities include a log scale of space, in which one log unit of physical distance corresponds to one unit distance of psychological space. Euclidean refers to the metric of space in which distance across two dimensions is computed by the familiar Euclidean formula. That is, two points, $(x 1, y 1)$ and $(x 2, y 2)$, in two-dimensional space have the distance square root ([ $\mid x 1-$ $\left.x 2 \mid]^{2}+[|y 1-y 2|]^{2}\right)$ between them. Another alternative is the city block metric: $|x 1-x 2|+|y 1-y 2|$ (Shepard, 1987).

Wilkie's (1989) pigeons had the task of remembering one of nine locations in a square grid on a panel. The size and shape of the space were comparable to those faced by our pigeons. The locations were marked by keys, one of which lit up as a sample key for a trial. After offset of the sample key and a delay, all nine keys were lit. The bird's task was to peck the sample key for that trial. The data thus consisted of a $9 \times 9$ matrix of confusion errors. Multidimensional scaling on these data led Wilkie to the conclusion that the scale of space for pigeons was linear and Euclidean. The scaling by no means produced perfect fits, but Wilkie attributed unsystematic misfits to errors in the data.

With a number of generalization gradients with $\mathrm{S}+$ at different values, Shepard $(1958,1965,1987)$ used multidimensional scaling methods to extract both the underlying psychological scale and the shape of the generalization function. Shepard (1965) scaled the classic data of Guttman and Kalish (1956) on generalization across wavelengths of light in pigeons. The data consisted of several gradients with $\mathbf{S}+$ at different wavelengths. Each generalization function was scaled to a proportion of responding at $\mathrm{S}+$. With multidimensional scaling, the spacing between the wavelengths used in tests was then adjusted to make the different generalization curves as similar as possible. That is to say, when the curves are all translated so that the $S+$ values coincide, they should deviate from one another as little as possible. This adjustment process is constrained because the same value along the dimension of generalization appears in several curves. Thus, one curve cannot be adjusted without affecting others. The common shape after the multidimensional scaling was taken to be the shape of the generalization function, and the scale that delivered this was taken to be the psychological scale.

For mechanistic and functional reasons, Shepard (1958, 1987) predicted that the shape of the generalization function would be exponential over the psychological scale. That is, $y=\exp (-k x)$, where $y$ represents amount of responding as a proportion of responding at $\mathrm{S}+, k$ is a scaling parameter, and $x$ measures psychological distance between a stimulus and $S+$, which can be extracted in the fashion indicated in the previous paragraph. Shepard (1987) presented 12 sets of data with exponential psychological scales, but for some sets of data (Church \& Gibbon, 1982; Nosofsky, 1986), a Gaussian shape $\left(y=\exp \left[-k x^{2}\right]\right)$ fit better.

Our data allowed us to examine the form of the generaliza- 
tion function, but not the metric (Euclidean vs. city block). The data came from, at most, two S+ locations so that multidimensional scaling in the style of Shepard (1965) could not be used. From Wilkie's (1989) conclusions, we took the underlying psychological scale to be linear and fitted both exponential and Gaussian curves to the data. A linear scale of space means that no transformation of the physical scale is needed at all: A unit of physical distance corresponds to a unit of psychological distance.

\section{Method}

The four birds from Experiment 2 (50-pixel spacing) and the four birds from Experiment 3 (30-pixel spacing) served in this experiment. The apparatus and stimuli were the same as in the preceding experiments. Birds in the 50-pixel spacing condition were given eight vertical generalization test sessions, each of which contained 84 baseline trials ( $42 \mathrm{~S}+$ and $42 \mathrm{~S}-$ ) and 21 test trials. On test trials, the stimulus was always presented in the $\mathbf{S}+$ location horizontally, but the vertical location varied across seven values: $50,100,150,200,250,300$, and 350 pixels from the top of the screen ( 200 was the baseline value). Each test value was presented three times in each test session. Birds in the 30-pixel spacing condition were given 16 sessions of vertical generalization sessions, each of which contained 72 baseline trials (36 S+ and 36 $S-)$ and 27 test trials. On half of these test sessions, the horizontal location of the stimulus on test trials was at the $S+$, whereas, on the other half of these test sessions, the horizontal test location was at the $\mathbf{S}-$. The test sessions at $\mathbf{S}+$ and $\mathbf{S}-$ alternated. In both cases, the vertical location varied across nine values: $120,150,180,210$, $240,270,300,330$, and 360 pixels from the top of the screen $(240$ was the baseline value). Each test value was presented three times in each test session. The contingencies on baseline and test trials were the same as those described in previous experiments.

\section{Results}

The response rates on the vertical series are shown in Figure 5. Although excitatory gradients were found around $\mathrm{S}+$, no noticeable gradient, excitatory or inhibitory, was found around $S-$. Block $\times$ Location ANOVAs confirmed these impressions. No statistical effects of any kind were found for the gradient around $S-$. For the vertical series around $\mathrm{S}+$ with 50-pixel spacing, significant effects of block, $F(3,9)=3.04$, location, $F(6,18)=4.68$, and Block $\times$ Location interaction, $F(18,54)=2.41$, were found. The interaction resulted mostly from the first block, which showed a wider generalization function than the other blocks. Taking data from Blocks 2-4 only, the interaction disappeared. In the vertical series around $S+$ with 30-pixel spacing, the ANOVA found only a location main effect, $F(6$, 18) $=5.35$, indicating stable data across blocks.

A symmetry test conducted on the data from all blocks in the vertical series around $S+$ with 50-pixel spacing revealed significant effects of block, $F(3,9)=9.94$, and distance from $S+, F(2,6)=14.56$, but no interaction, thus indicating symmetric results. The symmetry test on the vertical series around $\mathrm{S}+$ with 30-pixel spacing found a main effect of side, $F(1,3)=22.25$, and distance from $S+, F(2,6)=$ 13.90 , but no interactions. We have no ready interpretation
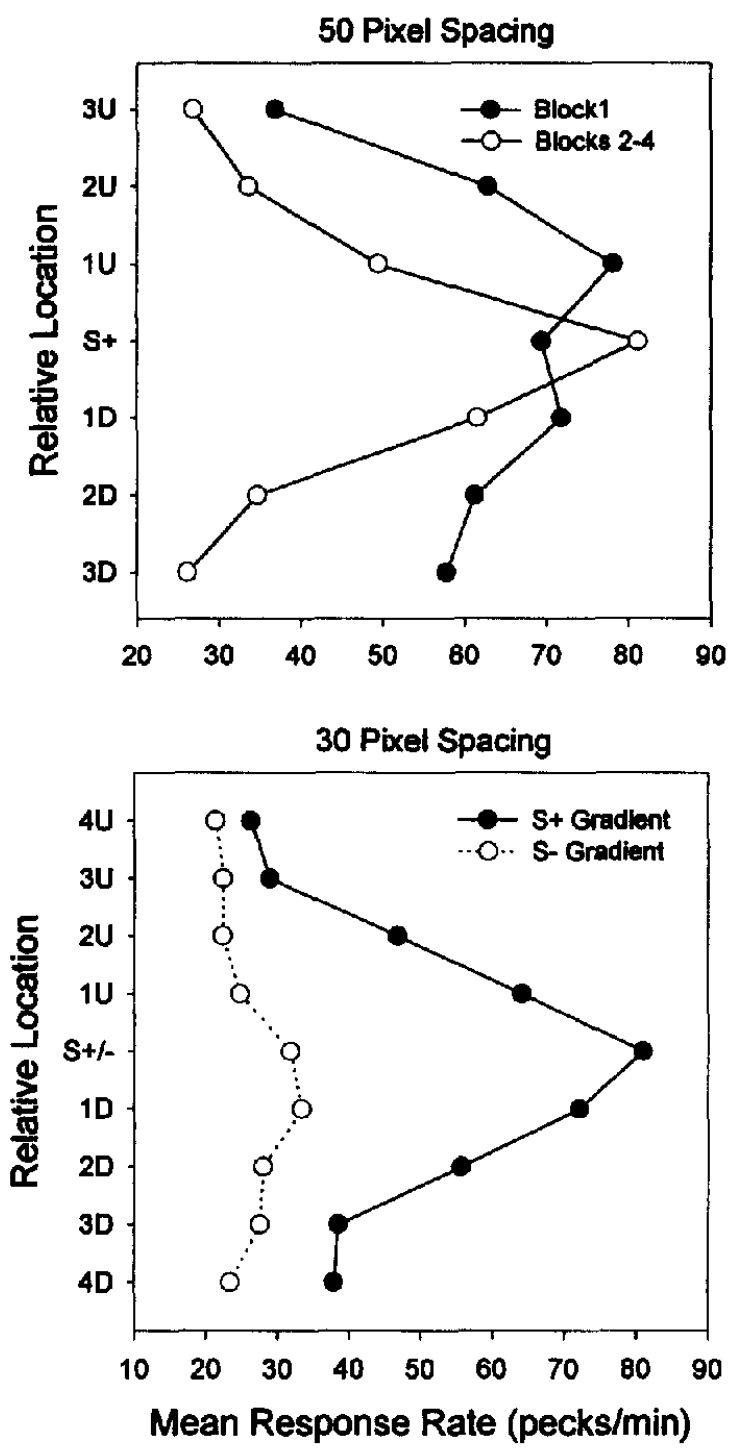

Figure 5. Response rates as a function of vertical location in Experiment 4. Top panel: 50-pixel spacing between locations. Bottom panel: 30-pixel spacing between locations. $U$ stands for up; D stands for down.

for the side main effect showing asymmetry. It might be a Type I error.

To determine whether the vertical gradients differed in form from the previously obtained horizontal gradients, we compared each vertical gradient around $\mathbf{S}+$ with its corresponding horizontal gradient. For birds tested with 50-pixel spacing, we first averaged the relative response rates at values equally distant from the $\mathbf{S}+$ on either side, thus giving single-sided generalization curves for each series in terms of rates relative to responding at $\mathrm{S}+$. We then compared Blocks 2-4 of the vertical series with Series 1 (Blocks 3-4) of horizontal testing in Experiment 2. The ANOVA excluded $\mathbf{S}+$ and thus consisted of two series and three distances from $\mathbf{S}+$. Only a main effect of distance was found, $F(2,6)=27.18$, indicating a similar shape for the 
vertical and horizontal dimensions. In Experiment 3, it was not clear if the peak-shift effect ever subsided completely. We thus compared Series 3 (the $S$ - side, all blocks) of Experiment 3 with the vertical series around $\mathrm{S}+$ with 30-pixel spacing. The upward and downward gradients of the vertical series were kept separate. The ANOVA again excluded $\mathbf{S}+$ and thus had three gradients by four distances from $\mathrm{S}+$. It also revealed only a distance main effect, $F(3$, $9)=19.44$. Thus, the vertical and horizontal gradients were similar. This suggests the same scaling of space along the two dimensions.

Gaussian and exponential best fits were calculated on a linear scale of space for three gradients: (a) Series 1 of Experiment 1, Blocks 2-4; (b) vertical series around $S+$ with 50-pixel spacing, Blocks 2-4; and (c) vertical series around $\mathrm{S}+$ with 30-pixel spacing, all blocks. Data were coded relative to the rate at $\mathrm{S}+$ in each block and were averaged across blocks and sides. For the third gradient, we also fitted each side separately. Both showed a similar pattern of results to the curve with both sides averaged so that only the latter is presented. Each fitted curve had one free parameter, the scaling parameter $k$ that adjusts the rate at which the curve falls off. A unit distance was 50 pixels. The equation for the exponential curve was $y=\exp (-k x)$; for the Gaussian curve, it was $y=\exp \left(-k x^{2}\right)$. The parameter $k$ was estimated to the third decimal to minimize the sum of absolute deviations between data and theoretical curves.

Curve-fitting results are shown in Figure 6, and parameter values and average misfits are listed in Table 1 . The exponential shape approximates the generalization function found in the vertical series, whereas the Gaussian function best describes the function from Experiment 1. Unexpectedly then, the scaling results across experiments differed, and we have added to the conflicting results found for the shapes of generalization functions.

We compared the generalization gradient from Experiment 1 with the vertical series with 50-pixel spacing, both of these series having the same spacing between locations. The data on relative rates that went into the curves of Figure 6 were subjected to a split-plot ANOVA with $\mathbf{S}+$ excluded. The outermost location from Experiment 1 was also excluded. Factors were thus two series (between subjects) and three distances from $S+$ (within subject). The ubiquitous distance main effect surfaced again, $F(2,6)=88.03$, but a Series $\times$ Distance interaction was also found, $F(2,6)=$ 5.29 , indicating that the two curves differed in shape.

\section{Discussion}

The fact that two spatial generalization gradients turned out exponential in shape on a linear scale supports at once both the linear scale and Shepard's law (Shepard, 1987). Both the scale and the form followed from theory. Caution, of course, should be taken with these preliminary results. First, the data came from only two $S+$ locations, and the replicability of results across locations should systematically be examined. Second, one of the curves did not come out exponential on a linear scale.

The Gaussian shape found for the generalization gradient

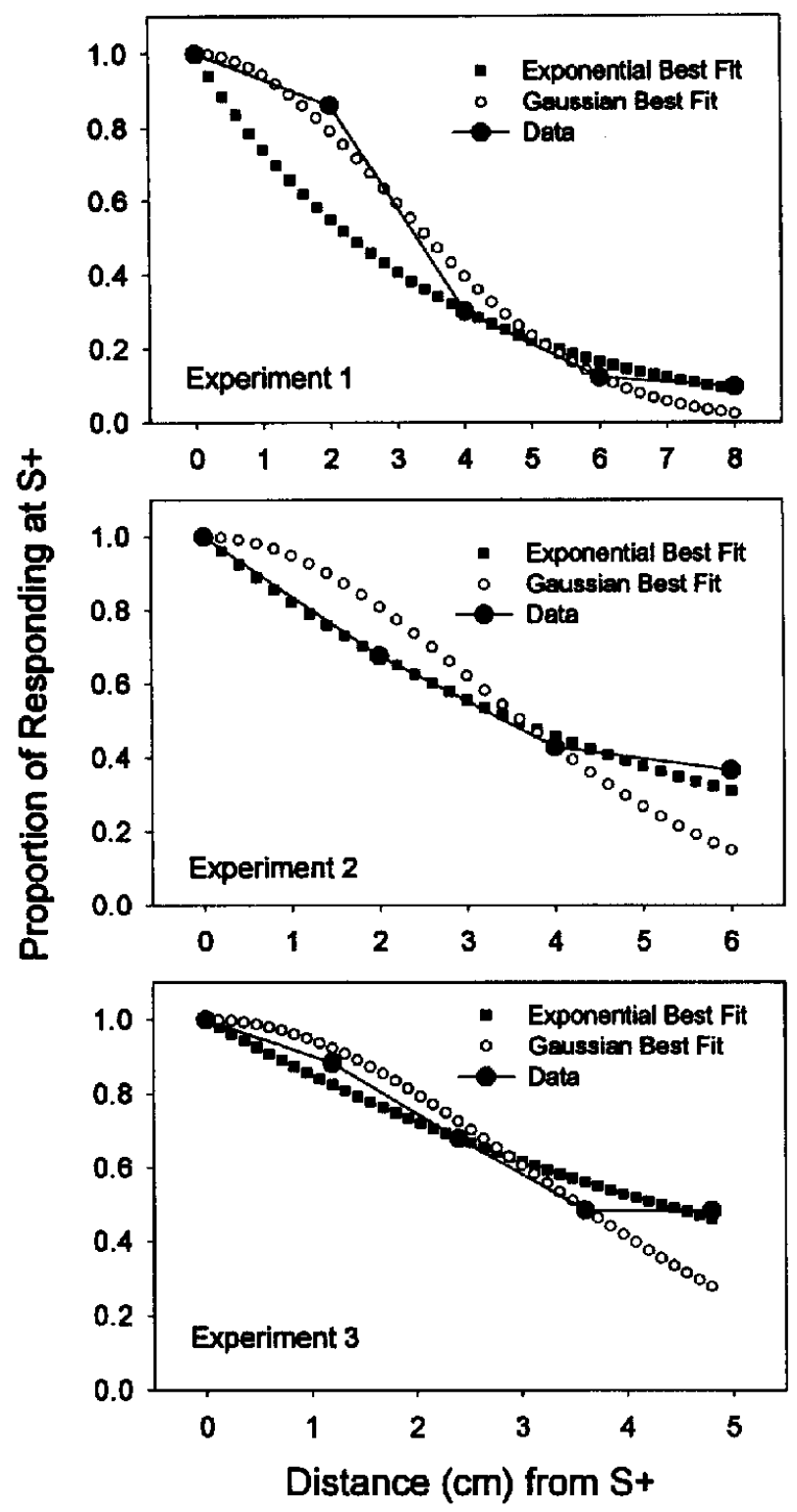

Figure 6. Curve fits of data from Experiments 1 (horizontal), 2 (50-pixel vertical spacing), and 3 (30-pixel vertical spacing). Response rates are expressed as a proportion of response rate at $S+$. Theoretical fits are of exponential $(y=\exp [-k x])$ and Gaussian $\left(y=\exp \left[-k x^{2}\right]\right)$ forms.

from Experiment 1 might come about either because the underlying scale of space is different or because the form of the gradient is different. We favor the latter interpretation. We can find no theoretical justification to argue that the psychological scale of space should differ across the experiments. On the other hand, both exponential and Gaussian shapes have been found in generalization curves. Nosofsky (1986) found the Gaussian shape for human generalization over orientations and size. Cheng and Wearden (1996) examined generalization gradients across temporal durations. For humans, the gradients were Gaussian in shape 
Table 1

Parameter Value and Average Absolute Deviation per Data Point for Exponential and Gaussian Fits of Spatial

Generalization: Experiments 1-3

\begin{tabular}{cccccc}
\hline & \multicolumn{2}{c}{ Exponential fit } & & \multicolumn{2}{c}{ Gaussian fit } \\
\cline { 2 - 3 } \cline { 5 - 6 } Experiment & $k$ & $\begin{array}{c}\text { Average } \\
\text { deviation }\end{array}$ & $k$ & $\begin{array}{c}\text { Average } \\
\text { deviation }\end{array}$ \\
\hline 1 & 0.600 & 0.073 & & 0.232 & 0.048 \\
2 & 0.390 & 0.021 & & 0.211 & 0.087 \\
3 & 0.322 & 0.031 & & 0.133 & 0.059 \\
\hline
\end{tabular}

Note. Curves were fitted on a linear scale of distance with 50 pixels defined arbitrarily as a unit of distance.

over a log scale of duration. For rats, data from Church and Gibbon (1982) were Gaussian in shape over a linear scale of duration.

What might have led to the different forms for the gradients from our pigeons? Nothing in the previous history of the birds in each experiment seems relevant so that the experimental manipulations are implicated. One possible difference is vertical versus horizontal dimensions, but we find this unlikely. Theoretically, this is an unwieldy interpretation. Empirically, the horizontal generalization curves at asymptote resemble their vertical counterparts in Experiments 2 and 3. Order effects also are a possibility that we cannot rule out because the vertical gradients in Experiments 2 and 3 were obtained after generalization testing in the horizontal continuum. We think it is more likely, however, that the differences reflect the training protocols used in the two experiments. The major difference that comes to mind is the larger number and proportion of $S$ - trials used in Experiments 2 and 3. If this interpretation is correct, then the $\mathrm{S}$ - training produces more than peak-shift effects. It can also affect the shape of the generalization function, even, as in the case of Experiment 2, after the short-lived peak shift has subsided. The effect of training at one $S-$ location must also transfer to other locations to affect the shape of the generalization function.

It is encouraging to find a factor within the same set of experiments that affects the shape of the generalization function. In discussions of Nosofsky's (1986) results (Ennis, 1988; Nosofsky, 1988; Shepard, 1986, 1988), a key theoretical distinction concerning the generalization task was made. The task was said to contain two components: (a) distinguishing stimuli perceptually and (b), for discriminable stimuli, generalizing, that is, assigning the stimulus to the same category as S+ or not. Shepard's exponential law is supposed to apply to the second component but not to the first. Where distinguishing stimuli is a significant problem, Gaussian gradients are likely. What is unclear is how to distinguish these two components without circular appeal to the form of the generalization gradient obtained. Further investigations of empirical factors that affect the form of the gradient are crucial in this enterprise. Training with $S-$ needs to be systematically manipulated, within the same experiment and preferably within subject. The issue of the psychological metric of space should also be investigated in a generalization paradigm, in which horizontal, vertical, and diagonal axes are used for obtaining generalization curves.

\section{General Discussion}

The results of peak-shift manipulations can be summarized as follows: When the $S-$ was $4 \mathrm{~cm}$ away from $S+$, an area shift was found early in testing. The $S+$ side had higher rates of responding than the $\mathrm{S}-$ side, but the peak of responding was at $S+$. This asymmetry was short lived: From Test Session 5 on, the distribution looked roughly symmetric about $S+$. When the $S-$ was $2.4 \mathrm{~cm}$ away from $\mathrm{S}+$, its effects were larger. A peak shift was obtained early in testing. This subsided to an area shift, but asymmetry was still found at the end of testing. The amount and durability of spatial peak shift thus depend on the distance between $\mathrm{S}+$ and $\mathbf{S}^{-}$.

The scaling of generalization gradients showed two patterns. When trained with only an S+ (Experiment 1), the gradient was approximately Gaussian over a linear scale of space. When trained with both $\mathrm{S}+$ and $\mathrm{S}-$, the gradient was approximately exponential over a linear scale of space, supporting Shepard's law (Shepard, 1987). This shape was found for the vertical dimension, which was orthogonal to the horizontal dimension along which the $S-$ was found. We attributed this to the effects of training with $S$ - rather than vertical versus horizontal dimensions.

\section{Peak Shift}

To the best of our knowledge, this is the first demonstration of peak shift in the spatial domain in any species. Moreover, the pattern of results is analogous to peak shift in other domains, in that the closer the psychological distance between $\mathrm{S}+$ and $\mathrm{S}-$, the larger the effect. Thus, it appears that stimulus control by the location of a stimulus is similar to control by properties of the stimulus itself. Recently, an unpublished experiment on spatial peak shift in honeybees was conducted (Cheng, 1996). The insects landed on a sponge $(10 \times 7 \mathrm{~cm})$ on which was placed a small capful of sugar water. On $\mathbf{S}+$ trials, the sponge was directly in front of a bottle serving as a landmark. On S - trials, the sponge was displaced by $10 \mathrm{~cm}$, and tap water replaced sugar water. An $S$ - trial lasted $1 \mathrm{~min}$ from the time the bee first flew over the sponge. It was then followed by an $\mathrm{S}+$ trial. This was necessary for practical reasons because the forager will not fly home without obtaining her fill of sugar water. No effect of the S- training was detectable: The generalization gradient on unrewarded tests remained symmetrical throughout. The bees received small numbers of training trials in comparison with pigeons. The tasks differed in many other ways. It is premature to claim species differences, but this provocative difference in results warrants further investigation.

We did not find any range effects in our experiments. None were expected, as the birds received only occasional testing mixed with continued training. The continued training should anchor the adaptation level should the creatures 
be using adaptation levels. Our manipulations were aimed at controlling the effects of adaptation levels and should not be seen as evidence against Thomas's (1974) adaptation-level hypothesis. What we have found is that spatial peak shift can be found even when adaptation level is controlled for. Some other mechanism than comparison with adaptation level must be producing our peak shift here. We discuss one mechanism after considering generalization gradients.

Recently, we have also conducted experiments on temporal peak shift in pigeons (Spetch \& Cheng, in press). In these experiments, the temporal duration of a stimulus was the dimension varied. $\mathrm{S}+$ was one duration, and $\mathrm{S}-$ was another. The generalization function after peak-shift training was a step function. Durations on the $\mathrm{S}+$ side had high pecking rates, durations on the $\mathbf{S}-$ side had low pecking rates, and the intermediate duration had an intermediate rate.

\section{Generalization}

The spatial generalization gradients from our pigeons showed either a Gaussian or an exponential decay on a linear scale of space. Recent data have been obtained on honeybees as well (Cheng, 1997). The setup in these experiments was like the experiments on peak shift in honeybees. The target was in front of a bottle serving as a landmark. Occasionally, the target was moved to another location for an unrewarded test. Across experiments, what served as the target, and hence what was moved on tests, was manipulated to create different salience levels of the target relative to all other unmoved landmarks. Clearly, the more salient the target, the less the generalization decrement should be, a pattern confirmed in the data. The shapes of the gradients were well fitted by exponential functions, but, interestingly, the scale was not linear. Instead, the scale had to be based on the psychology of how bees use landmarks.

Honeybees are known to use the distance and compass direction from goal to landmarks in landmark-based search (Cartwright \& Collett, 1982, 1983; Cheng, Collett, Pickhard, \& Wehner, 1987; Cheng, Collett, \& Wehner, 1986; Collett \& Baron, 1994). In laboratory situations, Cartwright and Collett $(1982,1983)$ found that distance judgment was based largely on the retinal size projected by a landmark. The psychological scale of distance for bees thus might be based on a combination of discrepancies in compass direction and retinal projection, discrepancies between the $S+$ location and the test location. Such a theoretical scale delivered the exponential shape of spatial generalization gradients for all experiments, without the need for any multidimensional scaling whatsoever. The only free parameter was the relative weighting of compass direction to retinal size. The parameter of the best fits was virtually identical to the corresponding parameter in a model of landmark-based search in honeybees (Cartwright \& Collett, 1982). The metric over the two dimensions (discrepancy in projected size and discrepancy in compass direction) could not be ascertained, as city block and Euclidean metrics produced similar scales. These data, the first spatial generalization gradients from an invertebrate, thus strongly support both the model of landmark use in bees and Shepard's law (Shepard, 1987).

\section{Modeling}

Shepard's (1958) mechanistic reason for the exponential law invokes the notion of diffusion over time across psychological space. Imagine a topographically arranged array of units, each unit corresponding to a point in psychological space. Think of $S+$ training as activating one of these points. Over time, however, the activation spreads. Because S+ trials occur repeatedly, the activation pattern of units consists of an average of functions of different degrees of spread. Shepard argued that such an average should produce something close to an exponential shape. Staddon and Reid (1990) formulated a more recent version of this model as well to account for generalization.

We attempted some versions of diffusion models of our making to account for the area shift found in Series 1 of Experiment 2. The attempt was by no means exhaustive. Many features of models may be modified, and, without further constraints, an exhaustive search is hardly worthwhile. However, it is instructive to see how one such model works.

The psychological space for the model was a rectangular grid including all tested locations vertically and horizontally. Locations are defined absolutely in the model and not relatively. Neighboring units were 10 pixels apart. We modeled $S+$ training as feeding the value 1 to the unit corresponding to $\mathrm{S}+. \mathrm{S}-$ training consisted of feeding the value 0 to the $S-$ unit. We did these two things together in one step. Next came the diffusion process, in which the fed values spread to neighbors. We did this by a smoothing process often used by scientists on data: Each unit's value was averaged with those of a number of neighbors. We chose square grids of neighbors for averaging, not for any theoretical reason but because it was practical on the spreadsheet (Excel 5). Averaging over a $5 \times 5$ grid produced the best results, and we report those. Each step in the model then consisted of feeding in values to $S+$ and $S-$ units and then of smoothing.

We iterated steps in the model in 50-step units until we found a pattern most resembling the first block of data. In calculating resemblance, we relativized all measures (model and data) as a proportion of the value at $\mathrm{S}+$ and looked for the minimum sum of absolute deviations between model and data. Step 950 produced the best fit (Figure 7).

We then started giving tests to the model starting at Step 950. The same smoothing process occurred in a step of testing, but we fed in different values. Tests were modeled as feeding zero into all tested locations. In one step then, 1 was fed into $\mathrm{S}+$, and 0 was fed into all other test locations. The zeros on the $\mathbf{S}+$ side lowered the unit strengths on that side. In 270 steps (nearest 10-step unit), we found the best fit of the model to the data of Blocks 3 and 4 averaged (Figure 7). In both cases, the model produced gradients resembling exponential functions.

We were not successful in modeling the peak-shift data from Experiment 3. Various versions of this averaging model failed to produce peak shift but, rather, produced only area shift. The asymptotic training pattern for these models (i.e., the gradient after many steps of training) resembles a 
Fit of Model to Data from Experiment 2, Series 1

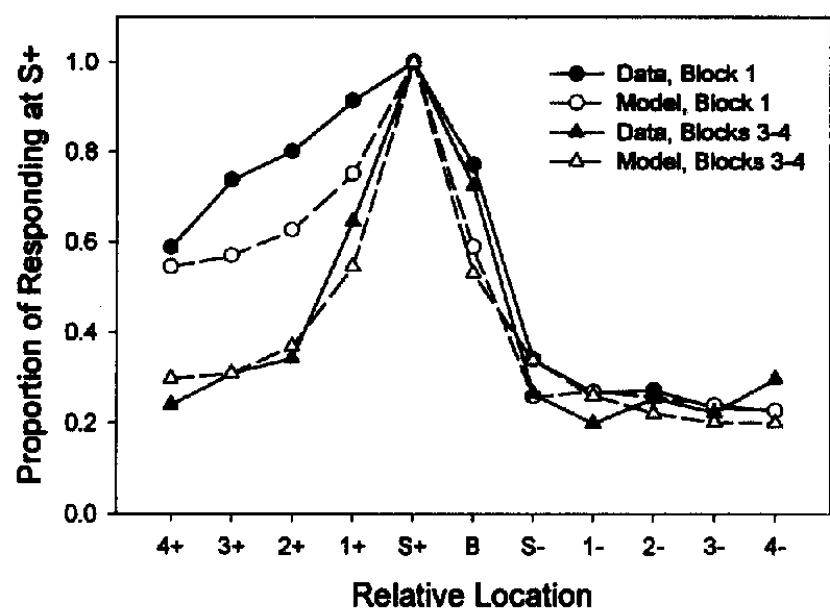

Figure 7. Curve fits of averaging model to the data from Series 1 of Experiment 2. B indicates location between the $S+$ and $S-$.

step function, with the $S+$ side at a high level, the $S-$ side at a low level, and the location in between $S+$ and $S-$ at an intermediate level. $S+$ is slightly elevated, and $S-$ slightly depressed, reminding one of a figure for illustrating lateral inhibition.

\section{Conclusions}

These experiments, conducted on a touch-screen task with pigeons, provide the first demonstration of peak and area shifts in the spatial domain. The closer the $S$ - was to the $\mathrm{S}+$, the larger and longer lasting the effect. Over trials of testing, the effect diminished. In examining spatial generalization gradients, both a Gaussian shape and an exponential shape were found over a linear scale of space. We suspect that training with $\mathrm{S}$ - trials affects the shape of generalization gradients, but the issue needs much more empirical and theoretical analysis.

\section{References}

Ames, L. L., \& Yarczower, M. (1965). Some effects of wavelength discrimination on stimulus generalization in the goldfish. Psychonomic Science, 3, 311-312.

Bloomfield, T. M. (1967). A peak shift on a line-tilt continuum. Journal of the Experimental Analysis of Behavior, 10, 361-366.

Cartwright, B. A., \& Collett, T. S. (1982). How honeybees use landmarks to guide their return to a food source. Nature, 295, 560-564.

Cartwright, B. A., \& Collett, T. S. (1983). Landmark learning in bees. Journal of Comparative Physiology A, 151, 521-543.

Cheng, K. (1986). A purely geometric module in the rat's spatial representation. Cognition, 23, 149-178.

Cheng, K. (1992). Three psychophysical principles in the processing of spatial and temporal information. In W. K. Honig \& J. G. Fetterman (Eds.), Cognitive aspects of stimulus control (pp. 69-88). Hillsdale, NJ: Erlbaum.

Cheng, K. (1996). [Spatial peak shift in honeybees]. Unpublished raw data.
Cheng, K. (1997). [Spatial generalization in honeybees confirms Shepard's law]. Unpublished raw data.

Cheng, K., Collett, T. S., Pickhard, A., \& Wehner, R. (1987). The use of visual landmarks by honeybees: Bees weight landmarks according to their distance from the goal. Journal of Comparative Physiology A, 161, 469-475.

Cheng, K., Collett, T. S., \& Wehner, R. (1986). Honeybees learn the colour of landmarks. Journal of Comparative Physiology A, 159, 69-73.

Cheng, K., \& Spetch, M. L. (in press). Mechanisms of landmark use in mammals and birds. In S. Healy (Ed.), Spatial representation in animals. London: Oxford University Press.

Cheng, K., \& Wearden, J. H. (1996). [The form of temporal generalization gradients]. Unpublished raw data.

Church, R. M., \& Gibbon, J. (1982). Temporal generalization. Journal of Experimental Psychology: Animal Behavior Processes, 8, 165-186.

Collett, T. S., \& Baron, J. (1994). Biological compasses and the coordinate frame of landmark memories in honeybees. Nature, $368,137-140$.

Doll, T. J., \& Thomas, D. R. (1967). Effects of discrimination training on stimulus generalization for human subjects. Journal of Experimental Psychology, 75, 508-512.

Dougherty, D. M., \& Lewis, P. (1991). Stimulus generalization, discrimination learning, and peak shift in horses. Journal of the Experimental Analysis of Behavior, 56, 97-104.

Ennis, D. M. (1988). Confusable and discriminable stimuli: Comment on Nosofsky (1986) and Shepard (1986). Journal of Experimental Psychology: General, 117, 408-411.

Ernst, A. J., Engberg, L., \& Thomas, D. R. (1971). On the form of stimulus generalization curves for visual intensity. Journal of the Experimental Analysis of Behavior, 16, 177-180.

Gallistel, C. R. (1990). The organization of learning. Cambridge, MA: MIT Press.

Guttman, N., \& Kalish, H. I. (1956). Discriminability and stimulus generalization. Journal of Experimental Psychology, 51, 79-88.

Hanson, H. M. (1959). Effects of discrimination training on stimulus generalization. Journal of Experimental Psychology, $58,321-334$.

Helson, H. (1964). Adaptation level theory. New York: Harper \& Row.

Honig, W. K., \& Stewart, K. E. (1993). Relative numerosity as a dimension of stimulus control: The peak shift. Animal Learning and Behavior, 21, 346-354.

Honig, W. K., \& Urcuioli, P. J. (1981). The legacy of Guttman and Kalish (1956): Twenty-five years of research on stimulus generalization. Journal of the Experimental Analysis of Behavior, 36, 405-445.

Jenkins, H. M., \& Harrison, R. H. (1960). Effect of discrimination training on auditory generalization. Journal of Experimental Psychology, 59, 246-253.

Nosofsky, R. M. (1986). Attention, similarity and the identificationcategorization relationship. Journal of Experimental Psychology: General, 115, 39-57.

Nosofsky, R. M. (1988). On exemplar-based exemplar representations: Reply to Ennis (1988). Journal of Experimental Psychology: General, 117, 412-414.

Purtle, R. B. (1973). Peak shift: A review. Psychological Bulletin, $80,408-421$.

Riccio, D. C., Urda, M., \& Thomas, D. R. (1966). Stimulus control in pigeons based on proprioceptive stimuli from floor inclination. Science, 153, 434-436.

Rilling, M. (1977). Stimulus control and inhibitory processes. In W. K. Honig \& J. E. R. Staddon (Eds.), Handbook of operant behavior (pp. 432-480). Englewood Cliffs, NJ: Prentice Hall. 
Rudolph, R. L., \& Honig, W. K. (1972). Effects of monochromatic rearing on spectral discrimination learning and the peak shift in chicks. Joumal of the Experimental Analysis of Behavior, 17, 107-111.

Shepard, R. N. (1958). Stimulus and response generalization: Deduction of the generalization gradient from a trace model. Psychological Review, 65, 242-256.

Shepard, R. N. (1965). Approximation to uniform gradients of generalization by monotone transformations of scale. In D. I. Mostofsky (Ed.), Stimulus generalization (pp. 94-110). Stanford, CA: Stanford University Press.

Shepard, R. N. (1986). Discrimination and generalization in identification and classification: Comment on Nosofsky. Journal of Experimental Psychology: General, 115, 58-61.

Shepard, R. N. (1987). Toward a universal law of generalization for psychological science. Science, 237, 1317-1323.

Shepard, R. N. (1988). Time and distance in generalization and discrimination: Reply to Ennis (1988). Journal of Experimental Psychology: General, 117, 415-416.

Spence, K. W. (1937). The differential response of animals to stimuli differing within a single dimension. Psychological Review, 44, 430-444.

Spetch, M. L. (1995). Overshadowing in landmark learning: Touch-screen studies with pigeons and humans. Journal of Experimental Psychology: Animal Behavior Processes, 21, 166181.
Spetch, M. L., \& Cheng, K. (in press). A step function in pigeons' temporal generalization in the peak shift task. Animal Learning \& Behavior.

Staddon, J. E. R., \& Reid, A. K. (1990). On the dynamics of generalization. Psychological Review, 97, 576-578.

Thomas, D. R. (1974). The role of adaptation-level in stimulus generalization. In G. H. Bower (Ed.), The psychology of learning and motivation (Vol. 8, pp. 91-145). San Diego, CA: Academic Press.

Thomas, D. R., Mood, K., Morrison, S., \& Wiertelak, E. (1991). Peak shift revisited: A test of alternative interpretations. Journal of Experimental Psychology: Animal Behavior Processes, 17, $130-140$

Thomas, D. R., \& Setzer, J. (1972). Stimulus generalization gradients for auditory intensity in rats and guinea pigs. Psychonomic Science, 28, 22-24.

Wilkie, D. M. (1989). Evidence that pigeons represent Euclidean properties of space. Journal of Experimental Psychology: Animal Behavior Processes, 15, 114-123.

Received November 12, 1996

Revision received February 3, 1997

Accepted February 13, 1997 